\title{
Evaluation of fusion methods for GF-6 aiming to water body observation
}

\author{
YiZhe Wang ${ }^{1}$, Guo $\mathrm{Liu}^{2,3, *}$, Bai Xue ${ }^{1}, \mathrm{Li} \mathrm{Guo}^{1}$ and XueLi Zhang ${ }^{4}$ \\ ${ }^{1}$ Land Satellite Remote Sensing Application Center, Ministry of Natural Resources, Beijing, China \\ ${ }^{2}$ National Engineering Research Center for Geographic Information System, China University of Geosciences (Wuhan), Wuhan, China \\ ${ }^{3}$ Geoscience Documentation Center of China Geological Survey, Beijing, China \\ ${ }^{4}$ East China Mineral Exploration and Development Bureau, Nanjing, China
}

\begin{abstract}
Gaofen-6 (GF-6) has the advantages of wide coverage, multiple resolutions, and multiple bands, and can provide richer information for remote sensing interpretation. Image fusion method is the key process in high resolution remote sensing application. Guangdong-Hong Kong-Macao Greater Bay Area was selected as experiment area and four image fusion methods of HPF (High-Pass Fliter), NND (Nearest Neighbor Diffusion), GS (Gram-Schmidt) and Pansharp were employed to process panchromatic and multispectral imaging. In order to evaluate the result performances, firstly, four kinds of fusion results were evaluated by visual, and then established mean, standard deviation, entropy, average gradient, correlation coefficient and spectral distortion for quantitative evaluation of fusion results. The results demonstrated that The Pansharp algorithm and the GS algorithm have the best comprehensive evaluation of the GF-6 satellite fusion effect, taking into account the color effect of the image and the enhancement of spatial details, which can meet most fusion requirements. For land-based fusion areas, the Pansharp fusion method can be selected, and the GS fusion method can better reflect the water body information and is the most suitable fusion method for GF-6 satellite water body information enhancement among the four algorithms.
\end{abstract}

\section{Introduction}

In recent years, China's satellite observation application system has been gradually improved, and satellite data acquisition capabilities have been greatly improved. In 2010, a major special project of high-resolution earth observation system was formally implemented, and a perfect ending was achieved in 2020. China has formed a space-based earth observation capability with high spatial resolution, high time resolution, and high spectral resolution. It greatly meets the needs of my country's highresolution remote sensing data application. With the rapid development of high-resolution remote sensing satellites, how to make full use of the spatial and spectral information of remote sensing images has become an important issue in the processing and application of highresolution remote sensing. Fusion of high spatial resolution panchromatic images and low spatial resolution multispectral images can effectively superimpose spatial detail information and color spectrum information, which is a feasible way to solve this problem.

To evaluate the fusion methods of different feature types on the remote sensing images, researchers have done more algorithm comparisons one after another. Zhou Weifeng et al. used WorldView-2 images as the data source, and the Xiangshan Port in Zhejiang Province as the research area, compared Principal Component Analysis (PCA), Gram-schmidt (GS), NND, and Brovey transformation, Wavelet Transform 5 fusion methods for subjective qualitative and objective quantitative evaluation and analysis and found the method of PCA is the most suitable fusion method for coastal oyster farming remote sensing applications [1]. Using GF-1 and GF-2 images as data sources, Chen Jiwei et al. evaluated Brovey transformation, GS, NND and PCA four fusion methods. Results show that the NND method is best for the GF-1 images and the GS method is best for the GF-2 images on waveform fidelity [2]. Du Yong et al. used the three images of WorldView-2 as the data source to evaluate the fusion method. The results indicate that GS is the ideal fusing method in this study. The detailed information of suspended sediment transport is constructed using the fused images [3]. Guan Yuqing et al. chooses domestic GF-1 satellite $2 \mathrm{~m}$ full-color/ $8 \mathrm{~m}$ multi/spectral imagery to study the remote sensing image fusion method for enhancing cultivated land information. The results showed that when facing cultivated land information, the MHIS method was the best fusion image [4]. Lin Xue et al. based on the Pleiades-1 image, and the study found that the results of Pansharp and GS fusion have good visual effects, strong ability of spectral preservation with the correlation

\footnotetext{
*Corresponding author: liuguo@mail.cgs.gov.cn
} 
coefficient up to 0.8 . The two fusion algorithm images have their own advantages in the information extraction ability of different forest land levels. The Pansharp fusion result has a total classification accuracy of $86.55 \%$ at the forest land level, and the GS fusion result has the highest total classification accuracy of $78.66 \%$ at the forest type level. The selection of the specific fusion algorithm needs to be determined according to the information extraction level of its application [5]. Chen Chao et al. verified the applicability and effectiveness of four commonly used pixels level fusion algorithms, and found that the IHS transformation method distorted the spectral characteristics of the source image, so the feature spectra were easily to become vestigial; Wavelet transformation had superiority on maintaining spectral characteristics, but the results easily became block and blurred; PCA transformation maintained most detail texture and architectural feature of the source image, but it will lose some of the detail spectral information; the tasseled cap transformation method fusion results in clear edges of ground objects, but it is not for the subtle spectrum Weak information retention[6]. At present, a variety of remote sensing image fusion algorithms have been implemented in the mainstream commercial remote sensing software. Although they can improve the image spatial resolution better, for different sensor types, remote sensing algorithms have their own advantages and disadvantages, and there is no unified recognition fusing algorithm.

With the specific goal of facing urban water bodies, this paper analyzes the image fusion results of the Gaofen6 satellite (GF-6) to provide a basis for water remote sensing applications. The GF- 6 satellite has the advantages of high resolution, wide coverage, high quality imaging, high performance imaging, and high localization rate. It has been in operation for three years, and a large number of high-quality remote sensing images of Earth observation have been obtained, but there are few reports on the applicability of the GF- 6 satellite panchromatic and multispectral fusion algorithm. This paper selects GF-6 satellite images of the Guangdong-Hong Kong-Macao Greater Bay Area and uses four fusion methods of HPF, NND, GS and Pansharp to conduct fusion experiments, compare and evaluate visual and quantitative evaluation indicators, and analyze the applicability of the GF-6 satellite fusion method. The results can provide a reference for GF-6 satellites in the application of resource and environmental observation.

\section{Data and research area overview}

\subsection{Introduction of GF-6 satellite}

GF-6 is the sixth satellite of China's high-resolution earth observation system, developed by China Aerospace Science and Technology Corporation. It was successfully launched by Jiuquan Satellite Launch Center on June 2, 2018 by the Long March 2D rocket. On March 21, 2019, GF-5 and GF-6 were officially put into use, marking that the high-spectrum special-purpose hyperspectral capability has been formed. GF- 6 satellite carries a $2 \mathrm{~m}$ panchromatic / $8 \mathrm{~m}$ multispectral resolution camera with a width of $90 \mathrm{~km}$ and a $16 \mathrm{~m}$ multispectral resolution wide coverage camera with a width of $800 \mathrm{~km}$. It is a sunsynchronous orbit with a design life of 8 years. It is the first multi-spectral remote sensing satellite of china with red-edge spectral bands and domestic CMOS imaging devices.

GF-6 is a high-resolution optical satellite with high maneuverability in China's high-resolution groundobserving system that takes into account both census and detailed investigation capabilities. The satellite and GF-1 satellite network has realized the revisited observation of China's land area for 2 days, which greatly improves the scale and timeliness of the acquisition of remote sensing data, and effectively makes up for the existing domestic and foreign high-resolution multispectral satellite resources. And the self-sufficiency rate and application range of domestic remote sensing satellite data have been improved.

\subsection{Research area overview}

In this paper, domestic multi-spectrum PMS sensor of GF6 satellite (multi-spectrum $1 \sim 4$ band (resolution $8 \mathrm{~m}$ ) and panchromatic band (resolution $2 \mathrm{~m}$ ) are selected for fusion experiments. The experimental image date is September 18, 2019. The multi-spectral data and panchromatic data were spatially registered before the experiment.

The Guangdong-Hong Kong-Macao Greater Bay Area is a world-class urban agglomeration established by relying on the unique geographical location advantage of the "Bay", with outstanding advantages in marine resources. The study area is located in the waters of the Guangdong-Hong Kong-Macao Greater Bay Area, mainly consisting of three different types of waters. Area 1 is the waters near the artificial island of the Zhuhai-Macao Port, which is close to the city and contains more suspended sediment; Area 2 is the waters on both sides of the east entrance of the submarine tunnel, with strong water bodies; Area 3 is the fishery coastline waters, with aquaculture as a the Lord.

The study area has unique geomorphic conditions and complex water and sand conditions. It is also one of the waters with the densest coastal routes and the highest density of ships in my country and the world. In recent years, the marine ecological and environmental protection of the Guangdong-Hong Kong-Macao Greater Bay Area has faced tremendous challenges. Therefore, the comprehensive use of satellite remote sensing and marine monitoring and other technical means to build a coastline dynamic monitoring and monitoring network will help the Guangdong-Hong Kong-Macao Greater Bay Area to implement a comprehensive, Real-time, dynamic and three-dimensional monitoring is of great significance for promoting the sustainable development of the Guangdong-Hong Kong-Macao Greater Bay Area. 


\section{Fusion algorithm and effect evaluation method}

\subsection{Fusion algorithm}

(1) HPF (high-pass filtering fusion)

The HPF method is to perform a positive FFT transform on a multispectral image and replace the highfrequency part of the multispectral image after the FFT with a high-resolution panchromatic band image. Spectral fusion image [7]. The fusion effect is affected by the size of the filter. This paper chooses a high-pass filter window of $3 * 3$.

(2) NND (Nearest Neighbor Diffusion)

The HPF method down samples the panchromatic band to low resolution, performs linear regression on pixels in each band to obtain the contribution vector $\mathrm{T}$, and calculates the neighboring pixel difference factor using the original panchromatic band to generate the initial highresolution multi- Spectral images, and finally use $\mathrm{T}$ as the weight to normalize the spectrum [8].

\section{(3) GS (Gram-Schmidt)}

The GS method first uses spectral resampling to simulate the first component, transforms the multispectral image to orthogonal space, and replaces the first component with a high spatial resolution panchromatic image. Then obtain the fused image by inverse transform. The GS fusion algorithm has no band limitation on the image, and can fuse all multi-spectral bands in one fusion process [9].

\section{(4) Pansharp}

The Pansharp method uses the least squares method to best match the gray value relationship between the fusion of the original multispectral image, the panchromatic image and the fused multispectral image and the panchromatic image. And the use of mathematical statistics to improve the integration process automation. When applying this fusion method, it is best to designate all the multispectral bands as reference bands to reduce the deviation between the fused image and the original multispectral image [10].

\subsection{Fusion algorithm and effect evaluation method}

\subsubsection{Qualitative evaluation}

Qualitative evaluation mainly performs subjective evaluation from visual effects, that is, visual method. Through visual comparison of image spectrum, edges, texture and overall brightness before and after fusion, it can intuitively and conveniently reflect the spatial resolution and clarity of different fusion images. However, this evaluation method is relatively subjective, and is greatly affected by the observer's experience and technology, image types, application occasions, and environmental conditions, lacks stability, and does not guarantee the repeatability of the evaluation method.

\subsubsection{Quantitative evaluation}

Quantitative evaluation method is an objective method to quantitatively analyze images based on the statistical characteristics of the images. It can overcome the influence of human subjective factors and make scientific and objective evaluations of the performance of various image fusions for further research. Quantitative evaluation indicators are mainly divided into two categories, one is single-factor evaluation, including image mean, standard deviation, and information entropy; the other is comprehensive evaluation indicators, including average gradient, correlation coefficient, and relative deviation. According to the characteristics of GF-6 image, this experiment selects the mean value, standard deviation, information entropy and average gradient as the quality evaluation index of the fusion image, and quantitatively describes the spectral fidelity, information amount and image clarity of the fusion image.

(1) The mean is the average of all the pixel values in the image and reflects the average reflection intensity of the features.

$$
u=\frac{\sum_{i=1}^{M} \sum_{j=1}^{N} F(i, j)}{M N}
$$

$\mathrm{u}$ is the average value of the image, $F_{(i, j)}$ is the gray value at the position of the pixel (i, j), and $\mathrm{M}$ and $\mathrm{N}$ are the number of image rows and columns, respectively.

(2) The standard deviation reflects the discrete situation of the gray level of the image relative to the average gray level average value. It is generally believed that the larger the standard deviation, the more dispersed the gray level distribution, the better the separability between features, and the more image information.

$$
s t d=\sqrt{\frac{\sum_{i=1}^{M} \sum_{j=1}^{N}(F(i, j)-u)^{2}}{M N}}
$$

std is the standard deviation of a certain band of the image, $\mathrm{M}$ and $\mathrm{N}$ are the number of rows and columns of the image; $F_{(i, j)}$ is the gray value at the position of the pixel $(i, j) ; u$ is the average gray of the image after fusion.

(3) Information entropy reflects the richness of image information. The larger the information entropy, the more information the image contains.

$$
H=-\sum_{i} p(i) \log _{2}[p(i)]
$$

$\mathrm{H}$ is the information entropy of a certain band of the image, $p(i)$ is the probability of the pixel whose gray value is $i$; it is expressed by the ratio of the number of pixels with gray value $i$ and the total number of pixels in the image.

(4) The average gradient is the average value of the grayscale change rate of the image, which can reflect the detail expression ability of the image.

$$
G=\frac{1}{(M-1)(N-1)} \cdot \sum_{i=1}^{M-1} \sum_{j=1}^{N-1} \sqrt{\frac{\left[\frac{\partial F(i, j)}{\partial x}\right]^{2}+\left[\frac{\partial F(i, j)}{\partial y}\right]^{2}}{2}}
$$

$\mathrm{G}$ is the average gradient of a certain band of the image, $F_{(i, j)}$ is the gray value of the image at the point $(\mathrm{i}, \mathrm{j}), \partial \mathrm{F}$ $(i, j) / \partial x, \partial F(i, j) / \partial y$ are the partial derivatives of the image at the point (i,j) in the row and column directions.

(5) The correlation coefficient reflects the degree of preservation of the spectral information of the fused image. The larger the correlation coefficient, it means that the spectral features of the image before and after the fusion 
are approximately close and the information is maintained approximately well.

$$
\mathrm{C}=\frac{\sum_{i=1}^{M} \sum_{j=1}^{N}\left(\operatorname{Mul}_{(i, j)}-\overline{M u l}_{(i, j)}\right)\left(F_{(i, j)}-\bar{F}_{(i, j)}\right)}{\sqrt{\sum_{i=1}^{M} \sum_{j=1}^{N}\left(M_{(i, j)}-\overline{M u l}_{(i, j)}\right)^{2} \sum_{i=1}^{M} \sum_{j=1}^{N}\left(F_{(i, j)}-\bar{F}_{(i, j)}\right)^{2}}}
$$

$M u l_{(i, j)}$ is the gray value of the original multispectral image, $F_{(i, j)}$ is the gray value of the image after fusion.

(6) The degree of spectral distortion directly reflects the degree of spectral distortion of the fusion image and the original multi-spectral image quality inspection. The greater the spectral distortion, the smaller the fidelity, the smaller the distortion, the better the guarantee.

$$
\mathrm{D}=\frac{1}{M \times N} \sum_{i=1}^{M} \sum_{j=1}^{N}\left|F_{(i, j)}-M u l_{(l, j)}\right|
$$

$M u l_{(l, J)}$ is the gray value of the original multispectral image, $F_{(i, j)}$ is the gray value of the image after fusion.

\section{Results and analysis}

This paper selects the panchromatic image with a resolution of $2 \mathrm{~m}$ and the multispectral image with a resolution of $8 \mathrm{~m}$ from the Gaofen-6 satellite as experimental data. The study area selects three different types of waters near the Hong Kong-Zhuhai-Macao Bridge. Area 1 is the water area near the artificial island of the Zhuhai-Macao Port, which is close to the city, and has a large amount of suspended sediment; Area 2 is the water area on both sides of the east entrance and exit of the submarine tunnel, and the water body is highly fluid; Area 3 is the fishery shoreline waters, mainly aquaculture.

The geographic imaging accelerator GXL software is used to perform orthorectification and geometric registration of the image, and the correction error is within 1 pixel. Then HPF fusion, NND fusion, GS fusion, Pansharp fusion were used to carry out fusion experiments on the registered panchromatic and multispectral images. The fusion effect of each method is shown in Figure 1 3.

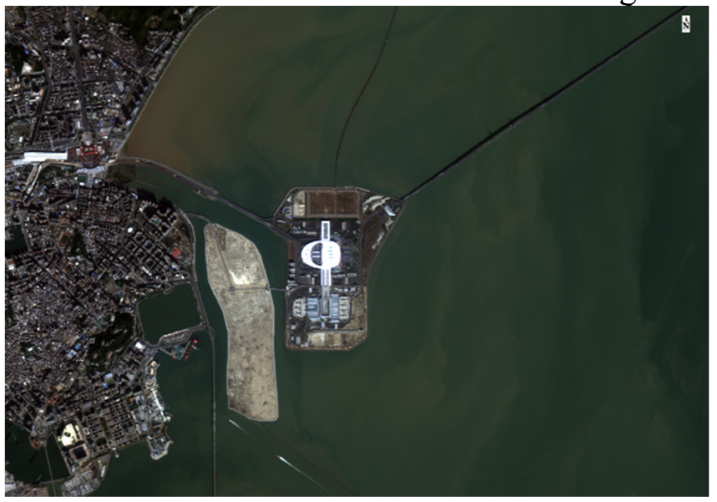

(a) Gram-Schmidt

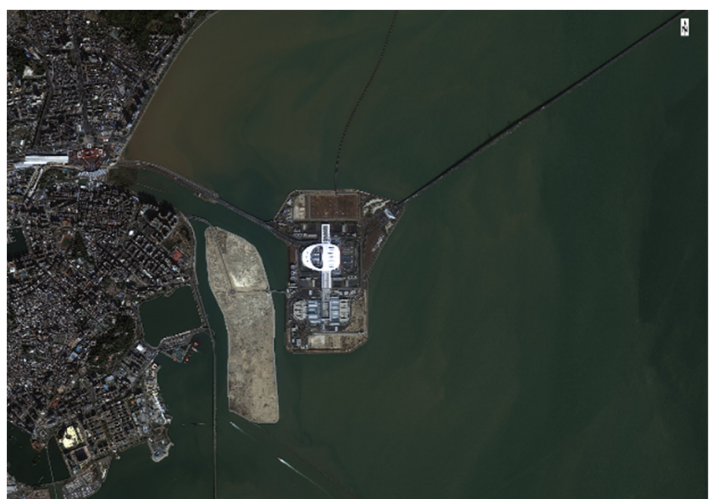

(b) HPF

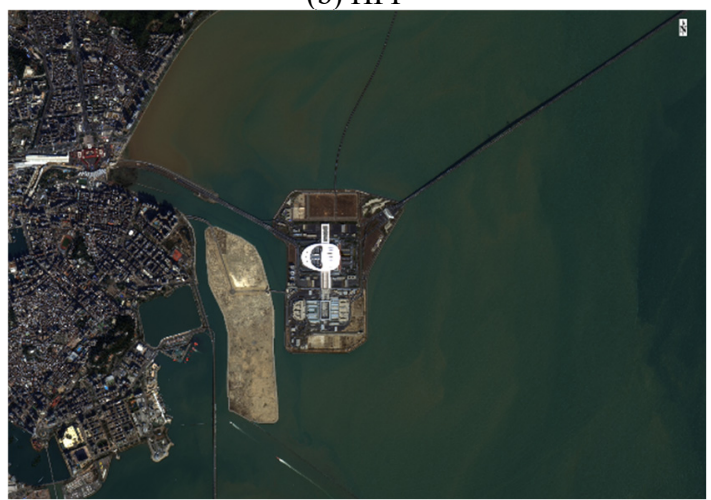

(c) NND

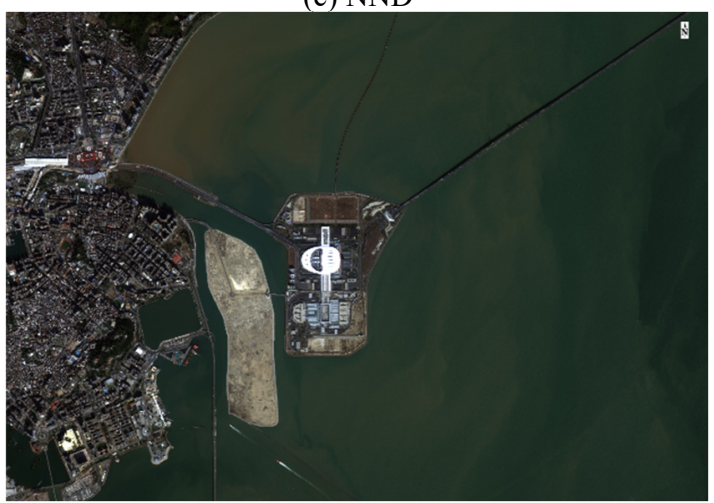

(d) Pansharp

Fig. 1. Comparison of 4 kinds of fusion method of study area 1

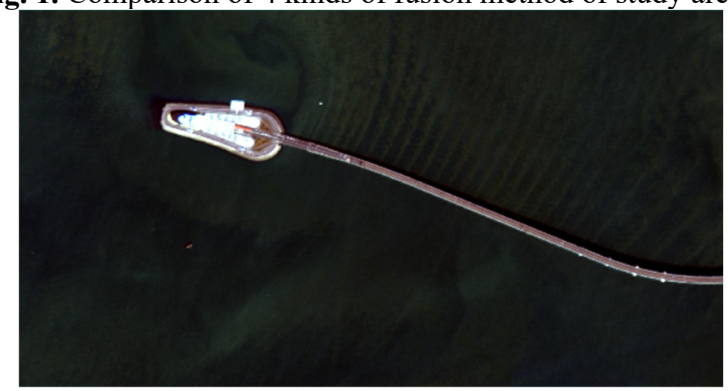

(a) Gram-Schmidt

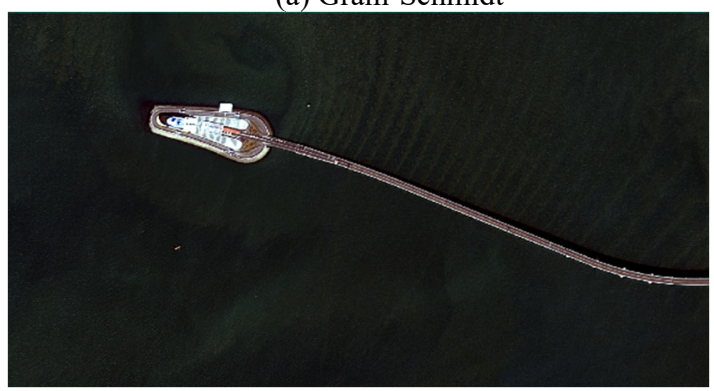

(b) HPF 


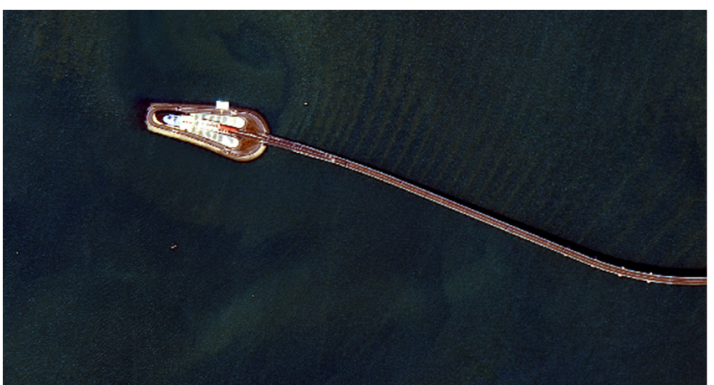

(c) NND

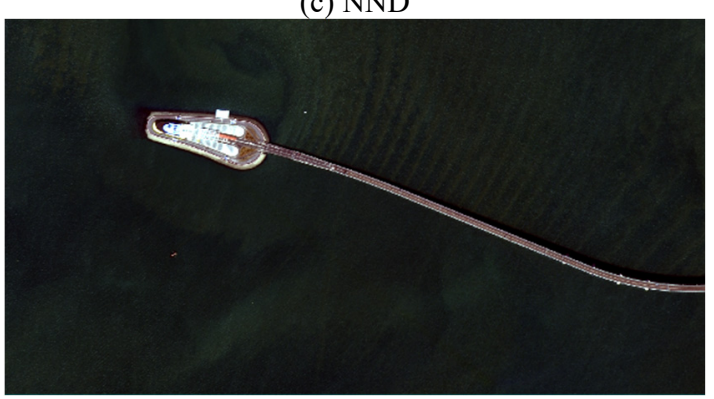

(d) Pansharp

Fig. 2. Comparison of 4 kinds of fusion method of study area 2

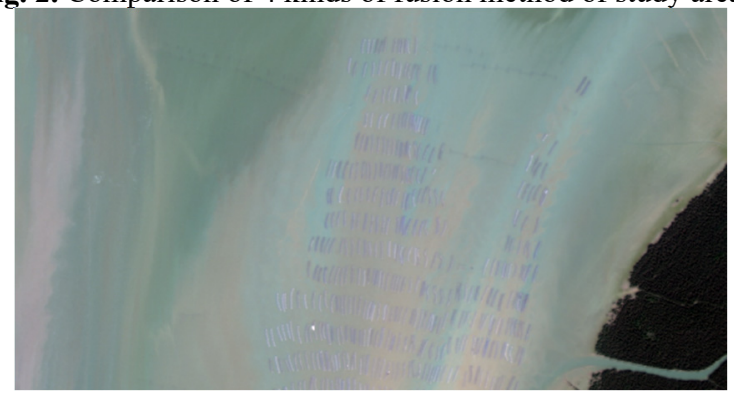

(a) Gram-Schmidt

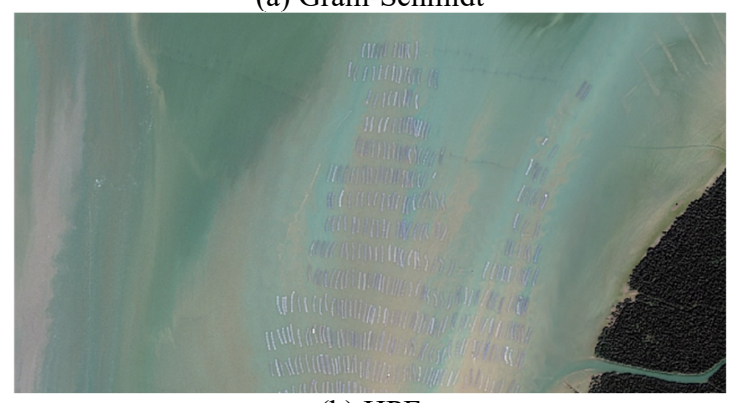

(b) HPF

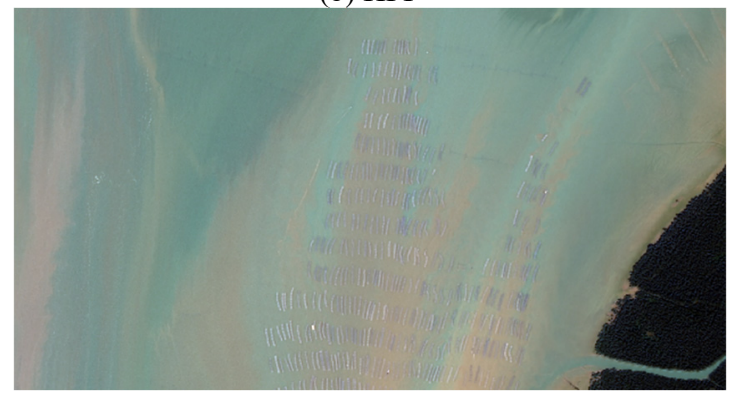

(c) NND

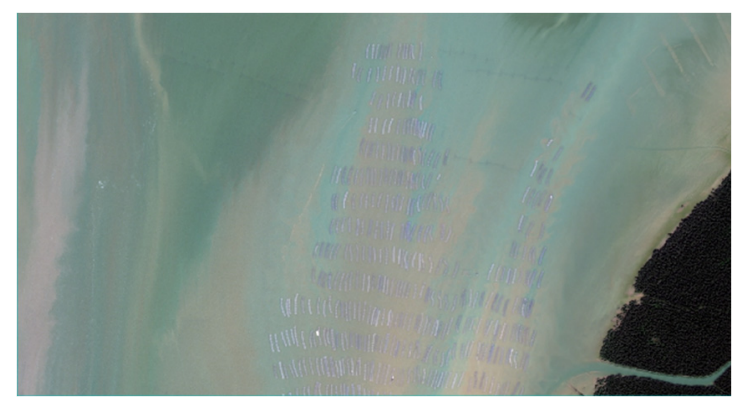

(d) Pansharp

Fig. 3. Comparison of 4 kinds of fusion method of study area 3

\subsection{Qualitative evaluation}

The visual interpretation of the fusion image shows that the four fusion algorithms have enhanced the resolution of the multispectral image by comparing Figures 1-3. Area 2 uses the NND algorithm to merge the construction land to be yellowish, and the color of the water body has a large deviation from the original MS image. The fusion result of GS algorithm is the closest to the multi-spectral MS image. However, from the perspective of details, the architectural part is overexposed and the detail texture is lost more. It is preliminarily judged that the NND algorithm is not suitable for the study of water bodies in area 2. Pansharp and HPF algorithm fusion image water body brightness is moderate, water body flowing ripples, road lanes are clear, while the spectral information of the original MS image is well preserved, which is suitable for region 2 water body fusion.

The colors of the results of the four fusion algorithms in area 1 are roughly the same as the original MS images. The suspended sediment texture information in the water bodies of Pansharp and GS algorithms is rich, and the boundaries of the buildings are distinct. The fusion result of the HPF algorithm has obvious graininess and a feeling of over-sharpening. After the NND algorithm is fused, the overall image is slightly blue. HPF and NND algorithms are obviously not suitable for area 1 fusion.

The overall effect of the four fusion algorithms in area 3 is not much different. From the enlarged view of the cultured floating raft, the fusion result of the GS algorithm and the original MS image have the highest retention in terms of image color. The second is the fusion result of Pansharp algorithm. The result of the fusion with the NND、 HPF algorithm is the biggest difference compared with the original MS image color. In terms of spatial resolution, Pansharp and GS algorithms have clear edges, moderate brightness, and distinct vegetation layers.

Therefore, the overall visual results show that Pansharp and GS algorithms have better fusion effects on the three regions in terms of spectrum preservation and spatial information enhancement.

\subsection{Quantitative evaluation}

In order to further compare the differences of several fusion algorithms, the mean, standard deviation, entropy, average gradient, correlation coefficient and spectral distortion of the four fusion results of the whole image, water image and excluded water image are respectively 
evaluated. The results are shown in Table 1.

Table 1. Objective evaluation results of the entire image

\begin{tabular}{|c|c|c|c|c|c|c|}
\hline & & MS & PSH & GS & NND & HPF \\
\hline \multirow{3}{*}{ Mean } & Image 1 & 730.73 & 721.69 & 734.52 & 784.14 & 716.73 \\
\hline & Image 2 & 653.04 & 651.43 & 654.34 & 736.82 & 650.34 \\
\hline & Image 3 & 676.90 & 674.88 & 682.24 & 741.45 & 670.72 \\
\hline \multirow{3}{*}{ Standard eviation } & Image 1 & 127.26 & 128.08 & 126.95 & 142.33 & 125.39 \\
\hline & Image 2 & 43.03 & 42.18 & 42.45 & 45.95 & 41.88 \\
\hline & Image 3 & 39.77 & 41.09 & 45.55 & 56.91 & 35.79 \\
\hline \multirow{3}{*}{ Entropy } & Image 1 & 7.53 & 7.49 & 7.56 & 7.69 & 7.53 \\
\hline & Image 2 & 4.76 & 5.16 & 4.76 & 6.28 & 5.19 \\
\hline & Image 3 & 6.40 & 6.50 & 6.48 & 6.91 & 6.46 \\
\hline \multirow{3}{*}{ Average gradient } & Image 1 & 5.46 & 10.64 & 5.79 & 15.88 & 11.88 \\
\hline & Image 2 & 1.31 & 3.41 & 1.45 & 7.48 & 3.38 \\
\hline & Image 3 & 0.82 & 2.73 & 1.08 & 4.62 & 2.94 \\
\hline \multirow{3}{*}{ Correlation coefficient } & Image 1 & & 0.955 & 0.988 & 0.876 & 0.910 \\
\hline & Image 2 & & 0.946 & 0.990 & 0.784 & 0.913 \\
\hline & Image 3 & & 0.982 & 0.996 & 0.930 & 0.952 \\
\hline \multirow{3}{*}{ Spectral distort-ion } & Image 1 & & 20.50 & 10.84 & 69.10 & 28.08 \\
\hline & Image 2 & & 5.81 & 2.60 & 85.62 & 7.04 \\
\hline & Image 3 & & 5.07 & 7.86 & 66.48 & 9.91 \\
\hline
\end{tabular}

Table 2. Objective evaluation results of land part

\begin{tabular}{|c|c|c|c|c|c|c|}
\hline & & MS & PSH & GS & NND & HPF \\
\hline \multirow{3}{*}{ Mean } & Image 1 & 238.08 & 234.37 & 238.32 & 248.34 & 230.57 \\
\hline & Image 2 & 27.51 & 27.10 & 27.55 & 28.78 & 26.87 \\
\hline & Image 3 & 63.42 & 62.59 & 61.46 & 63.75 & 64.69 \\
\hline \multirow{3}{*}{ Standard deviation } & Image 1 & 386.75 & 381.95 & 387.29 & 408.39 & 376.06 \\
\hline & Image 2 & 149.88 & 147.81 & 149.91 & 157.86 & 146.66 \\
\hline & Image 3 & 175.23 & 173.17 & 169.93 & 176.79 & 179.48 \\
\hline \multirow{3}{*}{ Entropy } & Image 1 & 3.60 & 3.60 & 3.61 & 3.68 & 3.63 \\
\hline & Image 2 & 0.51 & 0.51 & 0.50 & 0.53 & 0.52 \\
\hline & Image 3 & 1.10 & 2.11 & 1.18 & 1.39 & 1.36 \\
\hline \multirow{3}{*}{ Average gradient } & Image 1 & 5.95 & 9.68 & 6.07 & 13.11 & 11.16 \\
\hline & Image 2 & 1.47 & 1.62 & 1.34 & 1.90 & 1.65 \\
\hline & Image 3 & 1.14 & 2.11 & 1.38 & 2.67 & 2.49 \\
\hline \multirow{3}{*}{ Correlation coefficient } & Image 1 & & 0.994 & 0.997 & 0.986 & 0.988 \\
\hline & Image 2 & & 0.987 & 0.990 & 0.981 & 0.983 \\
\hline & Image 3 & & 0.994 & 0.995 & 0.992 & 0.989 \\
\hline \multirow{3}{*}{ Spectral distortion } & Image 1 & & 14.94 & 7.72 & 26.30 & 21.61 \\
\hline & Image 2 & & 2.22 & 1.25 & 3.42 & 2.87 \\
\hline & Image 3 & & 2.70 & 2.57 & 4.12 & 4.50 \\
\hline
\end{tabular}

Table 3. Objective evaluation results of water part

\begin{tabular}{|c|c|c|c|c|c|c|}
\hline & & MS & PSH & GS & NND \\
\hline \multirow{2}{*}{ Mean } & Image 1 & 492.59 & 486.90 & 495.76 & 535.36 \\
\cline { 2 - 7 } & Image 2 & 623.14 & 622.05 & 624.50 & 705.75 \\
\hline
\end{tabular}




\begin{tabular}{|c|c|c|c|c|c|c|}
\hline & Image 3 & 637.19 & 635.46 & 643.46 & 701.11 & 630.14 \\
\hline \multirow{3}{*}{ Standard deviation } & Image 1 & 318.24 & 314.76 & 320.56 & 346.06 & 314.00 \\
\hline & Image 2 & 123.46 & 123.03 & 123.44 & 140.37 & 122.69 \\
\hline & Image 3 & 178.15 & 177.96 & 180.33 & 196.44 & 176.04 \\
\hline \multirow{3}{*}{ Entropy } & Image 1 & 5.15 & 5.12 & 5.19 & 5.22 & 5.10 \\
\hline & Image 2 & 4.49 & 4.89 & 4.49 & 6.06 & 4.93 \\
\hline & Image 3 & 6.06 & 6.04 & 6.09 & 6.15 & 5.97 \\
\hline \multirow{3}{*}{ Average gradient } & Image 1 & 1.86 & 3.27 & 5.15 & 2.08 & 3.21 \\
\hline & Image 2 & 3.04 & 4.67 & 8.51 & 3.01 & 4.52 \\
\hline & Image 3 & 2.07 & 3.38 & 4.96 & 2.15 & 3.30 \\
\hline \multirow{3}{*}{ Correlation coefficient } & Image 1 & & 0.996 & 0.996 & 0.995 & 0.997 \\
\hline & Image 2 & & 0.955 & 0.956 & 0.950 & 0.951 \\
\hline & Image 3 & & 0.993 & 0.993 & 0.990 & 0.990 \\
\hline \multirow{3}{*}{ Spectral distortion } & Image 1 & & 7.02 & 4.57 & 44.28 & 8.03 \\
\hline & Image 2 & & 6.12 & 3.96 & 84.50 & 6.96 \\
\hline & Image 3 & & 4.41 & 7.29 & 64.77 & 8.65 \\
\hline
\end{tabular}

By comparing the statistical data of the original multispectral image and the four fusion images in the table (1-3), the following conclusions are obtained.

(1) The four algorithms have little difference in the mean, standard deviation, and information entropy after the fusion of the entire image. When comparing the average gradients, the average gradient of the NND is the largest, followed by the PSH and the HPF, indicating that the three algorithms have better spatial details of the entire fused image. However, when comparing the correlation coefficient and the spectral distortion, it is found that the NND has the worst spectral fidelity effect. Taken together, the PSH algorithm has the best overall fusion effect among the five fusion methods.

(2) In the fusion results of land part, the mean standard deviation of the NND and GS is higher than that of the MS image, and the fusion result is brighter; the mean standard deviation of the PSH is slightly lower than that of the MS image, and the fusion result is darker. Information entropy and average gradient are important indicators for evaluating image information and spatial details. It can be seen from the table that the information entropy of the NND is the highest, indicating that the information amount of the fusion image has increased; the information entropy of the other four algorithms is close to that of the original MS image, and the information amount of the original image is well maintained. The average gradient of NND, PSH and HPF is significantly higher than the original MS image; the average gradient of NND is the largest, and the spatial detail enhancement effect is the best. The correlation coefficient and spectral distortion directly reflect the effect of maintaining the spectrum. The correlation coefficient between the NND and the original MS image is the smallest, the spectral distortion is the largest, and the fidelity of the spectrum is the worst.

(3) In the fusion results of water part, the NND algorithm has the largest mean standard deviation, the image brightness is overexposed, which is not conducive to observing the water information, and the spectral distortion is the largest. The NND algorithm is the least ideal water fusion algorithm. The GS algorithm has the smallest spectral distortion and the largest average gradient. It is the most suitable method for water fusion among the four methods.

\section{Conclusion}

Remote sensing image fusion is an effective method to improve image resolution, enhance image utilization, and fully mine image information. This paper takes water as the research area and uses GF-6 satellite data to perform data fusion using HPF, NND, GS, and Pansharp four fusion algorithms. On the basis of subjective evaluation, from the perspective of spectral fidelity, image clarity, and image information, six objective evaluation indexes were selected to evaluate the fusion result. The experimental results show that, Pansharp algorithm and GS algorithm perform well in enhancing spatial details; GS algorithm is better in maintaining spectral information; HPF algorithm fusion results are over-sharpened, the visual effect is not good, and it is easy to cause texture loss. The NND algorithm has a small correlation coefficient and the largest spectral distortion. Compared with the other three algorithms, the effect of the spectral consistency with the source image is poor.

Therefore, according to the three regional evaluation results of this study, the Pansharp algorithm and the GS algorithm have the best comprehensive evaluation of the GF-6 satellite fusion effect, taking into account the color effect of the image and the enhancement of spatial details, which can meet most fusion requirements. The Pansharp algorithm can be selected for the land-based fusion area, and the water-based fusion area GS algorithm can better reflect the water body information. However, in actual work, integration needs are diversified, and specific problems should be analyzed in detail, and appropriate integration methods should be selected. This research selected four classic and practical methods to conduct experiments and analysis based on GF-6 satellite data, 
which has certain guidance and reference significance for the future production, application and scientific research of GF-6 satellite data.

\section{References}

1. Zhou Weifeng, Cao Li, Li Xiaoshu. Assessments of fusion methods using WorldView-2 satellite images for coastal oyster culture observation $[\mathrm{J}]$. Remote Sensing Technology and Application, 103-109, 33(1) (2018)

2. Chen Jiwei, Shen Qian, Zhu Li. Influence of different fusion methods based on GF Images on the spectral fidelity of urban water [J]. Journal of Beijing Polytechnic University, 677-682, 43(5)( 2017)

3. Du Yong, Zhang Xiaoyu, Huang Dasong. Evaluation of fusion methods for Worldview-2 aiming to water body observation [J]. Journal of Zhejiang University (Engineering Science), 993-1000, 5 (2015)

4. Guan Yuqing, Mi Wentian. Research on GF-1 satellite remote sensing image fusion method for cultivated land [J]. Hubei Agricultural Sciences, 104-107,113, 5(2019)

5. Lin Xue, Peng Daoli, Huang Guosheng. Evaluation analysis of fusion algorithms for Pleiades-1 data oriented to extraction of forestland information $[\mathrm{J}]$. Engineering of Surveying and Mapping, 18-24, 25(11)( 2016)

6. Chen Chao, Qin Qiming, Wang Jinliang. Comparison of quality evaluation methods for image fusion of farmland remote sensing [J]. Transactions of the Chinese Society of Agricultural Engineering, 95-100, 10(2011)

7. Wei Jun, Li Bi-cheng. Remote-sensing image fusion based on ihs transform、wavelet transform and high pass filtering $[\mathrm{J}]$. Journal of Information Engineering University, 46-50, 4(2)( 2003)

8. Sun W, Messinger D. Nearest-neighbor diffusionbased pan-sharpening algorithm for spectral images[J].Optical Engineering, 013107, 53(1)( 2014)

9. Laben C A, Brower B V. Process for enhancing the spatial resolution of multispectral imagery using pan - sharpening: US, 6011875[P], (2000-01-04)

10. Pang Jinjiao, Sun Rui, Wang Wen. Influence of registration error on land cover classification and change detection in high-resolution remote sensing image[J] . Remote Sensing Technology and Application, 498-505, 29(03) (2014) 